\title{
Optimal monitoring for project- based emissions trading systems under incomplete enforcement
}

\section{Working Paper}

\section{Author(s):}

Ohndorf, Markus

Publication date:

2010

Permanent link:

https://doi.org/10.3929/ethz-a-006309790

Rights / license:

In Copyright - Non-Commercial Use Permitted

Originally published in:

IED working paper 13 
Optimal Monitoring for project-based Emissions Trading Systems under incomplete Enforcement

Markus Ohndorf 



\section{Optimal Monitoring for project-based Emissions Trading Systems under incomplete Enforcement}

\section{Markus Ohndorf}

ETH Zurich, Institute for Environmental Decisions (IED), Chair of Economics (ohndorfm@ethz.ch)

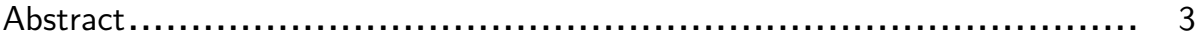

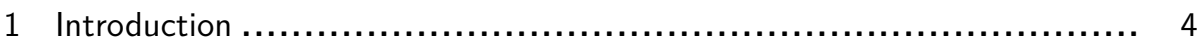

2 Incomplete Enforcement of Emission Reductions....................... 5

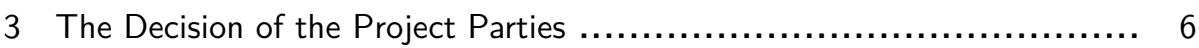

3.1 Decision under Full Enforcement .............................. 6

3.2 Decision under Incomplete Enforcement ........................ 7

4 Optimal Monitoring Policy with an unlimited Budget $\ldots \ldots \ldots \ldots \ldots \ldots \ldots \ldots \ldots$

5 Monitoring with a Limited Budget.................................... 13

5.1 Optimal Monitoring Pressure under a given Budget ................ 17

5.2 Regulation with Project Admission .............................. 20

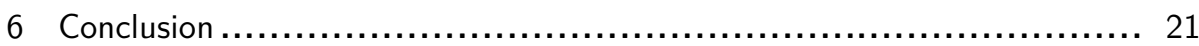

Mathematical Appendix $\ldots \ldots \ldots \ldots \ldots \ldots \ldots \ldots \ldots \ldots \ldots \ldots \ldots \ldots \ldots \ldots \ldots \ldots \ldots \ldots \ldots \ldots \ldots \ldots \ldots \ldots \ldots, 23$

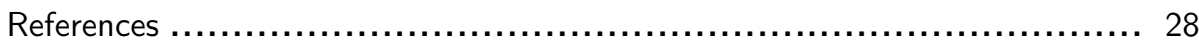

\section{Acknowledgments}

We gratefully acknowledge Soham Baksi, Fabienne Braune, Stefanie Engel, Renate Schubert, Moritz Rohling, and audiences at the Annual International Conference of the German Economics Association and the fourth World Congress of Environmental and Resource Economists (WCERE) 2010 for useful comments and suggestions on a previous draft of this paper. All remaining errors are our own. 


\section{Abstract}

Project-based Emissions Trading Schemes, like the Clean Development Mechanism, are particularly prone to problems of asymmetric information between the project parties and the regulator. Given the specificities of these schemes, the regulator's optimal monitoring strategy significantly differs from the one to be applied for capand-trade schemes or environmental taxes. In this paper, we extend the general framework on incomplete enforcement of policy instruments to reflect these specificities. The main focus of the analysis is to determine the regulator's optimal spot-check frequency under the plausible assumption that the submitted projects vary with respect to their verifiability. We find that, given a limited monitoring budget, the optimal monitoring strategy is discontinuous, featuring a jump within the set of projects with lower verifiability. In this region, actual abatement is low and can fall to zero. For these cases, the sign of the slope of the strategy function depends on the actual relationship of the abatement cost and the penalty function. We conclude that, in a real-world context, project admission should ultimately be based on the criterion of verifiability.

Keywords: environmental regulation, emissions trading systems, audits and compliance

JEL classification numbers: K32, D42, D82 


\section{Introduction}

Project-based emissions trading schemes, like the Clean Development Mechanism (CDM) under the Kyoto-Protocol, are particularly vulnerable to problems of incomplete enforcement. These problems arise due to information asymmetries between the regulator and the project participants, creating an incentive to overstate emission reductions. Hence, the overall abatement level is likely to be lower than predicted within neoclassical setups, where the optimal amount of emission reductions is achieved through market forces. Under the standard neoclassical assumptions the interaction of supply and demand leads to an equalization of marginal abatement costs and the certificate price. However, if the the above-mentioned information asymmetries are taken into account the level of abatement achievable through creditbased systems might be significantly lower.

Within this paper, we develop an analytical model of a credit-based system that takes potential overreporting of emission reductions into account. The main focus is laid here on the optimal decisions of the regulator, given the impossibility to fully enforce compliance to the Mechanism. Just as with any microeconomic model, the framework presented here is not a mapping of one-to-one real world situations. Its major objective is rather to identify the specific effects of the above-mentioned information asymmetries on the decisions of a rational benevolent regulator. ${ }^{1}$

Given the importance of enforcement for the effectiveness of environmental policies, considerations of incomplete enforcement of instruments has become an important research field within environmental economics. Early research on incomplete enforcement of environmental regulation has mainly focused on the comparison of emission taxes and pollution standards. The first formal model on this issue was developed in Harford (1978), which was extended in Harford (1987) to include selfreporting by firms. Within more recent research the analyses were extended to the comparative performance of the different environmental policy instruments under incomplete enforcement. Schmutzler and Goulder (1997) focus on the difference between emission taxes and output taxes. Macho-Stadler and Perez-Castrillo (2006) analyze the optimal enforcement policy in the context of per-unit emission taxes. Malik (1990), Keeler (1991), Macho-Stadler (2006), and Stranlund (2007) also include cap-and-trade programs in their analysis.

Yet, credit-based schemes seem to have been neglected in the literature so far. To our knowledge, there exists no formal model deriving optimal monitoring for such systems. This might be due to the fact that credit-based systems only recently gained in importance, which is, in turn, to a large extent a result of the successful implementation of the Kyoto markets. On the other hand, the non-consideration of credit-based systems in the literature is particularly unfortunate in light of the elevated potential for fraudulent misreporting within such schemes. In this paper we present an extension of the tax-based model developed in Macho-Stadler and Perez-Castrillo (2006), to reflect the specificities in incomplete enforcement under

\footnotetext{
${ }^{1}$ In the context of the Clean Development Mechanism, the role of such a regulator is taken up by the CDM Executive Board and its supporting panels. As a consequence, the decisions of the external verifiers - the Designated Operational Entities-are not explicitly modeled. This simplification is justifiable on the grounds that the CDM verification mechanism is not capable to fully deter opportunistic overreporting. This incomplete deterrence can be attributed to two different reasons. First, even a thorough verification might not reveal opportunistic behavior in reporting, as information asymmetries between project participants and the verifier could persist. Second, given the fact that the DOEs are remunerated by the project participants, potential collusion in reporting of emission reductions cannot be excluded.
} 
credit-based schemes. As it turns out, considering credit-based emissions trading instead of environmental taxes does significantly alter both, the analysis as well as the results. We find, that depending on the relationship between certificate prices and the penalty scheme, optimal monitoring will feature jumps and might not necessarily be always increasing over the degree of verifiability of the projects. Furthermore, the possibility to deny projects access to the scheme will, if based on the criterion of verifiability, significantly improve the performance of credit-based mechanisms.

The paper is structured as follows. In the following section the general problem of opportunistic false reporting credit-based systems is explained further. The presentation of the formal model starts with section 3 , where the abatement and reporting decisions of a rational project developer are derived. Section 4 presents the optimal monitoring policy of a regulator disposing of an unlimited budget. In section 5, optimal monitoring is analyzed under the more realistic assumption of a limited budget. The paper concludes with a discussion of the model results.

\section{Incomplete Enforcement of Emission Reductions}

The basic problem with any environmental regulation aiming at the reduction of emissions is that emitters can either understate their actual emissions or overstate the emission reductions achieved. Given that these levels are not fully observable to the regulator, emitters have an incentive to misreport these values. In the case of a per-unit tax on emissions, for example, the reported emissions represent the basis from which the overall tax burden for the regulated entity is derived. While a reduction of emissions is associated with abatement costs, fraudulent misreporting of emissions might represent an attractive alternative for reducing the amount of taxes paid. In a cap-and-trade emissions trading system, the situation is similar. Here, the emitter has an incentive to increase the difference between the stipulated emissions cap and the reported emissions. Given that emission permits have a positive market value, an increase in this difference augments, ceteris paribus, the benefits for net sellers and decreases the costs for net buyers. Again, emitters might be tempted to overreport actually achieved emission reductions, in order to maximize the amount of disposable permits.

The potential for fraudulent reporting is even higher in credit-based systems, like the Clean Development Mechanism. In such a system, the amount of certificates generated is determined by the difference between the emissions of a baseline emission scenario and the reported project emissions. Hence, a fraudulent manipulation is possible ex ante, through an overstatement of the baseline, as well as ex post, through an understatement of actual project emissions. For this reason, both determinants of generated certificates are subject to a scrutinized verification within the CDM project cycle. Yet, it is likely that information asymmetries between project parties and the regulator persist. The baseline, for example, should ideally represent the scenario based on what would have happened without the implementation of the project. Clearly, any such plans for the project site are private informationa priori unknowable to the regulator-and are not necessarily truthfully revealed within the Project Design Document. Note that this information asymmetry with respect to the baseline is also at the basis of the well-known discussion on the additionality within the CDM. In addition to this baseline problem, the possibility of underreporting the actual emissions persists, just as for the other environmental 
policy instruments mentioned above.

As a consequence of the problem of opportunistic misreporting, any environmental policy needs to include an enforcement mechanism to be effective. In general, such a mechanism involves the monitoring of actual emissions through the regulator and a sanctioning of discovered fraudulent misrepresentation. In a resourceconstrained world, however, full enforcement of environmental policies is unlikely. As monitoring of emitters is costly, the effectiveness of the enforcement mechanism is constrained by the budget available to the regulator. Under these circumstances, it is more efficient to increase the deterrence effect by increasing the expected sanction through a tightening of the penalty. ${ }^{2}$ However, even if the budget constraint of the regulator does not bite, there might be situations within which enforcement remains incomplete. This is the case if the information asymmetry between emitter and regulator is structured in such a way that the probability of discovering fraudulent misreporting is lower than one.

The framework presented below builds heavily on the model developed in MachoStadler and Perez-Castrillo (2006), who analyze incomplete enforcement in the context of emission taxes. However, the adjustments made for representing a creditbased system do significantly alter both, the analysis as well as the results. As will be explained below, the major difference between tax schemes and credit-based systems is the fact that the objective function of the regulator changes with a switch from one system to the other. As the received credits are used to offset emissions elsewhere, a regulator interested in environmental integrity has to minimize not only the emissions, but the overall overreported amount of emission reductions. This minimization is subject to the constraint that the project parties, in turn, minimize their costs. For this reason the subsequent outline of the model starts with deriving the decisions of the single projects.

\section{The Decision of the Project Parties}

In the following sections a scheme is considered within which private actors can submit emission reduction or absorption projects generating credits that can be used to offset emissions occurring at an emission source which is not associated with the project. This setup describes, hence, schemes like the CDM where the generated credits are sold on a primary or secondary carbon market.

\subsection{Decision under Full Enforcement}

Throughout the following pages it is assumed that there exists a continuum of abatement projects for each of which the respective project participants have invested in a specific abatement technology determining a minimum level $\underline{e}$ and a maximum level $\bar{e}$ of emission reductions achievable within the project, where $\underline{e}, \bar{e}>0$. Within these limits the project participants choose the actual level of reductions $e$; hence $e \in[\underline{e} ; \bar{e}] .^{3}$ This assumption implies that the respective project is already accepted within the credit-scheme, but can generate different levels of emission reductions,

\footnotetext{
${ }^{2}$ This argument is one of the main postulates derived from the economic theory of crime. See Becker (1968).

${ }^{3}$ For example, a project might involve a fuel-switch within a power plant from coal to natural gas. In this case the upper and lower level of project emissions are pre-determined by the actual base demand of electricity, the applied emission factor, and the plant's capacity.
} 
dependent on the abatement effort levels incurred. A relaxation of this assumption will be discussed in section 5.2. The above depicted set-up adequately describes a large range of project types. For example, the reductions generated through fuel switching, reforestation, or energy efficiency-projects are not only dependent on the initial project investment, but also on the efforts incurred to actually implement the project itself.

It can be reasonably assumed that emission reductions cannot be generated without costs. Hence, any level of emission reductions is associated with a respective amount of abatement costs. The abatement cost function of the project is denoted as $\mathrm{c}(\mathrm{e})$, with $c^{\prime}(e)>0, c^{\prime \prime}(e)>0$, for all $e \in[\underline{e} ; \bar{e}]$.

In order to establish a benchmark for the model results under incomplete enforcement, it is useful to first describe the decision of the project participants for the case where the actual project reductions are fully observable. In this case the project participants report the emission reductions that have actually occurred, i.e. $e$. The reported emission reductions are certified and can then be sold on the market for the price $p$, which is assumed to be constant and publicly known. In this case, the project participants will minimize the net costs $C(e)$ of the project as follows:

$$
\min _{e} C(e) \text {, with } C(e)=c(e)-p e
$$

Note that under the assumed full-information case, the firm reports exactly the emission reductions actually achieved, and receives thus exactly $e$ certificates, which can be sold on the market for the price $p$. Hence, net costs $C(e)$ consist of the costs of reducing the emissions $\mathrm{c}(\mathrm{e})$ and the reduction of overall costs through the revenue from selling the thusly generated certificates. From the first order condition associated with (1), which determines the optimal level of emission reduction, follows:

$$
c^{\prime}\left(e^{*}\right)=p
$$

Equation (2) represents the well-known result that under perfect competition with full information the marginal abatement costs will be equal to the certificate price. The resulting first-best optimal level of emission reductions is denoted by $e^{*}$. This level will be used as a benchmark for the following analysis.

\subsection{Decision under Incomplete Enforcement}

If incomplete enforcement is introduced within the above-described framework the project participants' decision might change. In case of information asymmetries between the regulator and the project developer, the latter can chose any level of emission reductions $\tilde{e}$ but report to have actually reduced more. Note that, with rational actors, reported emission reductions $z$ will never be less than the actual reductions $\tilde{e}$ as the value of the certificates is positive. It is also assumed that for all projects there exists a maximum level of plausible emission reductions $\bar{e}$, i.e. for reports above this level no certificates will be issued. In the context of the CDM, this upper limit can be interpreted as restrictions to plausible baseline emissions established by the CDM Executive Board. Thus, formally the reported emission reductions are defined as $z \in[\tilde{e}, \bar{e}]$.

In order to increase the incentive for truthful reporting, the regulator has the possibility to monitor the projects. The probability of being monitored for each project type is $\alpha$, with $\alpha \in[0 ; 1]$. It is assumed that this probability is known to the project 
participants. In an application to the Clean Development Mechanism, $\alpha$ would be determined by the expected frequency of 'spot-checks' on each project type by the CDM Executive Board. The different projects are assumed to differ with respect to their 'verifiability', which is expressed through a project-specific probability $\beta$. If $\beta$ is 1 and the regulator monitors the project he is capable to determine without further problems whether the firm has overreported or not. The closer $\beta$ is to 0 , the more improbable is the success of such an assessment. Hence, the intensity of the information asymmetry is assumed to vary across the different project types and might become large enough that a verification through the regulator does not necessarily lead to the discovery of misreporting. Assuming different levels of verifiability is quite plausible if it is kept in mind that for any given project overreporting can be the result of either a misstated baseline, underreported project reductions, or both. In order to be able to isolate the effects of such differences in verifiability, it is assumed in the following that this is the only variable in which projects differ. In all other parameters the different projects are treated as being identical.

In case overreporting is discovered the project participants are required to pay back the revenue from overreported reductions and in addition to pay a fine. The regulator is assumed to make the fine contingent on the 'magnitude of the lie' $x$, which is defined as $x=z-e$. The relationship between the overreported amount and the corresponding fine is determined by the legislator of the scheme and defined through the penalty function $\theta(x)$, with $\theta(0)=0, \theta^{\prime}(x)>0$, and $\theta^{\prime \prime}(x)>0$ for $x>0$. Note that in order to have a deterrence effect, this function is a priori known by the regulated project parties. The assumption that the penalty is convex in the magnitude of the offense is quite realistic, as it seems to be in line with legal practice under many different circumstances. ${ }^{4}$

With incomplete enforceability of truthful reports, the project participants' optimal decision problem is altered. Taking into account the assumptions on the expected penalty made above, the minimization problem for each project type is defined as:

$$
\begin{gathered}
\min _{e, z} C(e, z, \alpha, \beta), \\
\text { where } C(e, z, \alpha, \beta)=c(e)-p z+\alpha \beta p(z-e)+\alpha \beta \theta(z-e)
\end{gathered}
$$

It is hence taken into account within the cost function that in case overreporting is discovered, the project participants will have to give back the excess certificates and pay a fine according to the progressive penalty schedule $\theta$. The first order conditions for (3) are:

$$
\begin{gathered}
\frac{\partial C}{\partial e}=c^{\prime}(e)-\alpha \beta p-\alpha \beta \theta^{\prime}(z-e)=0 \\
\frac{\partial C}{\partial z}=-p+\alpha \beta p+\alpha \beta \theta^{\prime}(z-e)=0
\end{gathered}
$$

The solutions to these necessary conditions indeed determine a local minimum, as is proven in the mathematical appendix. It is however to be noted that the optimization program in (3) is, in fact, also constrained through the domains of the variables $z$ and $e$. Depending on the values for the overall probability of discovery, i.e. $\alpha \beta$, the project participant's optimization can hence result in a corner solution.

\footnotetext{
${ }^{4}$ The assumption of convex punishment is widely used in a large part of the literature on incomplete enforcement. See, for example, Harford (1978), Harford (1987), Sandmo (2002), Cremer and Gahvari (2002), and Macho-Stadler and Perez-Castrillo (2006).
} 
This is definitely the case if the probability of discovery is zero. In this case, the project participants would chose the maximum plausible amount for the reported emissions, i.e. $z=\bar{e}$, while actually choosing the minimum possible amount of emission reductions, i.e. $e=\underline{e}$. This result reflects a complete unenforceability of truthful reporting and can, in fact, only occur with either an inactive regulator or a complete lack of verifiability of the project.

For those cases where the probability of discovery is positive, the expected penalty in case of discovery provides an incentive to reduce the overreported amount $x$, which is defined as $x=z-e$. To achieve such a reduction in $x$ the project parties can either increase the amount of actual emission reductions, or decrease the amount of reported reductions, or both. In order to understand the optimization of the participants, it is to bear in mind that a reduction in $\mathrm{z}$ would entail a decrease in issued certificates by a corresponding amount. Hence, the 'cost' for reducing reported emissions is equal to the price of the certificates $p$. As a consequence, with increasing values of $\alpha \beta$, cost-minimizing project parties will always prefer to hold $z=\bar{e}$ and to rather increase their actual reductions $e$ as long as the marginal cost of abatement remain lower than the certificate price $p$. Consequently, a reduction in $z$ will only occur if e is reduced to the level $e^{*}$, i.e. the first best optimal reduction level which is implicitly defined through equation (2). Hence, only if $\alpha \beta$ are high enough to induce $e^{*}$ the level of $z$ chosen will be determined through condition (5). Finally, if the probability of discovery is high enough, the expected penalty will be large enough to completely deter opportunistic overreporting, such that $x=0$.

These intuitive considerations are expressed more formally within proposition 1 , also defining the respective thresholds for the monitoring probability. The proposition represents in fact an adaptation of the findings of Macho-Stadler and PerezCastrillo (2006) to the case of credit-based emissions trading.

Proposition 1. For a given price of certificate $p$, audit probability $\alpha$, a penalty function $\theta(x)$, and a level of overreporting $x=z-e$, the optimal emission and report decisions $\left(e^{0}, z^{0}\right)$ for the project of type $\beta$ are:

(a) If $\alpha \beta=0$, then $e^{0}=\underline{e}$ and $z^{0}=\bar{e}$.

(b) If $\alpha \beta \in] 0, \frac{p}{p+\theta^{\prime}\left(\bar{e}-e^{*}\right)}\left[\right.$, then $e^{0} \in\left(\underline{e}, e^{*}\right)$ and $z^{0}=\bar{e}$, with

$$
c_{e}\left(e^{0}\right)-\alpha \beta p-\alpha \beta \theta^{\prime}\left(\bar{e}-e^{0}\right)=0 .
$$

(c) If $\alpha \beta \in\left[\frac{p}{p+\theta^{\prime}\left(\bar{e}-e^{*}\right)}, \frac{p}{p+\theta^{\prime}(0)}\left[\right.\right.$, then $e^{0}=e^{*}$ and $\left.\left.z^{0} \in\right] e^{*}, \bar{e}\right]$ as defined by:

$$
-p+\alpha \beta p+\alpha \beta \theta^{\prime}\left(z^{0}-e^{*}\right)=0
$$

(d) If $\alpha \beta \geq \frac{p}{p+\theta^{\prime}(0)}$, then $e^{0}=e^{*}$ and $z^{0}=e^{*}$.

Proof: See mathematical Appendix.

Figure 1 depicts the changes in levels of actual abatement and of reported reductions chosen by the project parties for different levels of probability of being punished. As implied by the proposition, the probability features several thresholds 
leading to a change in cost minimizing behavior. The figure also depicts the probability ranges within which the cases $(a)$ to $(d)$ hold. As expressed within proposition 1 (a), a zero probability of being punished induces the reduction and reporting levels that maximize overreported emissions. Within range (b) the project participants prefer to increase the level of actual reductions, while holding $z$ at the maximum plausible level $\bar{e}$. With further increase of the probability of being discovered, the actual reductions are held at the level $e^{*}$ while the reported reductions are reduced, as depicted in field (c). With a high enough probability of punishment, the parties are completely deterred from overreporting, as expressed within proposition 1 (d).

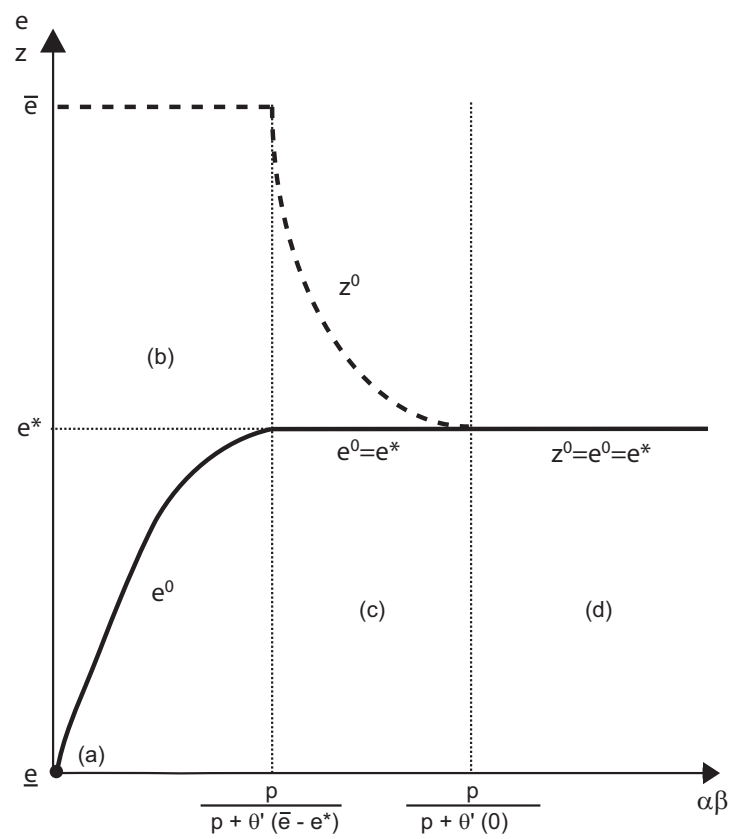

Figure 1: Firm's reported and actual emission reductions related to their monitoring probability

[Firm's reported and actual emission reductions]

\section{Optimal Monitoring Policy with an unlimited Bud- get}

Given the optimizing behavior of the project participants as derived above, a rational regulator needs to identify an optimal monitoring strategy. In order to model the monitoring decision with an existing project pool, the regulator is assumed to face a population of already registered projects. As explained before, the different projects are supposed to vary only with respect to their verifiability, which is parameterized by $\beta$. The project specific probability $\beta$ is distributed over the interval $[0,1]$ according to the density function $f(\beta)$, with $f(\beta)>0$ for all $\beta \in[0,1]$, and the cumulative function $F(\beta)$.

The objective of the enforcement agency is to minimize overall emissions, occurring within the regulatory scheme. When choosing the level of audit pressure 
for each project type, the regulator needs to account for the fact that credit-based systems fundamentally differ from other environmental policy instruments. Within cap-and-trade emissions trading or an emissions tax system, the only variable that is to be minimized are the actual emissions of the scheme's participants. Consequently, actors situated within area (c) of figure 1 would not require a further increase in the monitoring probability $\alpha$. For a credit-based system, like the Clean Development Mechanism, restricting enforcement on the actual emissions would not necessarily minimize overall emissions. Given that the credits generated through the projects are being used to offset emissions elsewhere, an over-issuance of such credits due to false reporting would lead to an undesired increase in global emissions compared to the full enforcement case. Hence, unlike other environmental policy instruments, credit-based systems require the regulator to minimize not only the actual reductions $e$ but also the reported reductions $z$.

Within the model presented here, optimal enforcement implies a minimization of overreporting, expressed through $x=z-e$, over the whole project population, parameterized by $\beta$. For simplicity, the cost of one unit of audit is normalized to one. To determine the optimal enforcement policy, the enforcement agency decides on auditing each type of firm, i.e. it choses $(\alpha(\beta))_{\beta \in[0,1]}$ in order to solve the following program.

$$
\begin{gathered}
\min \int_{0}^{1} z(\beta)-e(\beta) d \beta \\
\text { such that } \\
\int_{0}^{1} a(\beta) f(\beta) d \beta \leq B \\
\text { and } \\
e(\beta), z(\beta) \in \operatorname{argmin} C(\beta, \alpha ; e, z)
\end{gathered}
$$

Hence, to choose an optimal monitoring scheme, the agency needs to take into account its budget constraint (9), and the profit maximization of the single project participants, expressed through constraint (10). Within this section we only consider the second constraint to be binding.

As the choices of $z$ and $e$ for the different projects depend on the overall probability of being punished $\alpha \beta$, the optimal level of $\alpha$ varies for each project type according to its individual probability of verifiability $\beta$. Hence, taking the project parties' optimization into account, for each threshold identified in proposition 1, a specific value of $\alpha$ can be defined. First, we define:

$$
\hat{\alpha}(\beta) \equiv \frac{p}{\beta\left[p+\theta^{\prime}\left(\bar{e}-e^{*}\right)\right]}
$$

as the minimum probability that induces an emission level of $e^{*}$. As $\alpha, \beta \in[0,1]$, this probability level is only 'feasible' when $\hat{\alpha}(\beta) \leq 1$, i.e. $\beta \geq \hat{\beta}$, where:

$$
\hat{\beta} \equiv \frac{p}{\left[p+\theta^{\prime}\left(\bar{e}-e^{*}\right)\right]}
$$


A firm with $\beta<\hat{\beta}$ pollutes more than $e^{*}$ even at audit probability $\alpha=1$, since the probability of being discovered even if audited is very low.

Second, we define:

$$
\check{\alpha}(\beta) \equiv \frac{p}{\beta\left[p+\theta^{\prime}(0)\right]}
$$

as the minimum audit probability that induces a report $z=e=e^{*}$, i.e. truthful reporting. Again, this probability level is only 'feasible' if $\check{\alpha} \leq 1$, i.e. $\beta>\check{\beta}$, where:

$$
\check{\beta} \equiv \frac{p}{\left[p+\theta^{\prime}(0)\right]}
$$

Taking these different thresholds into account, it becomes clear that the assumed structure on the information asymmetries between regulator and project participants does not allow for the realization of the full information outcome. For low levels of $\beta$ even an audit probability of $\alpha=1$ is not sufficient to induce truthful reporting. Consequently, even with an unlimited budget the minimum global pollution level that an agency can achieve, is restricted by the informational constraints. This maximum level of global emission reductions induced by the credit-scheme given an unlimited budget can be defined as:

$$
e_{\beta}^{M A X} \equiv \int_{0}^{\hat{\beta}}\left(e^{* *}(\beta)-\bar{e}\right) f(\beta) d \beta+\int_{\hat{\beta}}^{\check{\beta}}\left(e^{*}-z^{* *}(\beta)\right) f(\beta) d \beta+[1-F(\check{\beta})] e^{*}
$$

where $e^{* *}$ and $z^{* *}$ are implicitly defined by

$$
\begin{gathered}
c_{e}\left(e^{* *}\right)-\alpha \beta p-\alpha \beta \theta^{\prime}\left(e^{* *}\right)=0, \text { for } \beta \in(0, \hat{\beta}] \\
\text { and } \\
-p+\alpha \beta p+\alpha \beta \theta^{\prime}\left(z^{* *}-e^{*}\right)=0, \text { for } \beta \in(\hat{\beta}, \check{\beta})
\end{gathered}
$$

The first term in (12) measures the overall impact on reductions of projects which will overpollute and overreport even if the monitoring probability $\alpha$ is equal to one. These projects are situated in field (b) of figure 1 . The second term expresses the impact on reductions of projects within field (c), achieving the optimal level of actual reductions but which can not be completely deterred from overreporting. The last term adds the reduction level of projects that are induced to fully comply to the terms of the mechanism.

The definition in (12) can be used to calculate the minimum budget necessary to achieve $e_{\beta}^{M A X}$. We denote this minimum budget by $\bar{B}$, which is defined through:

$$
\bar{B} \equiv F(\check{\beta})+\int_{\check{\beta}}^{1} \check{\alpha}(\beta) d \beta
$$

Proposition 2 immediately follows:

Proposition 2. When $B \geq \bar{B}$ the cost-minimizing agency sets an audit policy that satisfies $\alpha(\beta)=1$, for $\beta \in[0, \breve{\beta}[$ and $\alpha(\beta) \in[\check{\alpha}(\beta), 1]$ for $\beta \in[\check{\beta}, 1]$. 
Proposition 2 has an important implication for the design of audit policies for credit-based systems. In case such a scheme includes projects with large information asymmetries between project participants and the regulator, an increase in budget does not necessarily lead to a reduction in overreporting. As soon as the budget level $\bar{B}$ is reached, the marginal rate of deterrence equals zero. Hence, even if a maximization of reductions is the only objective of the agency, efficiency requires that the auditing budget of the regulator should be capped at $\bar{B}$.

\section{Monitoring with a Limited Budget}

In practice, it is likely that the number of audits which can be performed by the regulator is constrained by his budget. After all, budget $\bar{B}$ implies that all projects situated in regions (b) and (c) in figure 1 are audited with probability one. In the context of the Clean Development Mechanism, for example, this would mean that the Executive Board will carry out audits for projects with a lesser potential of verifiability with certainty. This would not be in line with the declared approach to carry out 'spot-checks' for project control which are by definition associated with a probability lower than one. It is hence realistic to assume that the budget constraint (9) of the regulator's optimization problem is binding. Thus, in the following it is assumed that $B \leq \bar{B}$.

Within the budget-constrained optimization, it is again to be taken into account that the regulator is not only interested in achieving an optimal level of actual reductions $e$-as would be the case for other policy instruments-but that the specificities of the credit-based approach require to take also the reported reductions $z$ into account. Again this is due to the fact that issued CDM certificates are used to offset emissions elsewhere. The regulator needs hence to decide for which project types an increase in spot-check frequency would result in the largest decrease in overall emissions. In this context, it is of particular importance whether the monitoring budget should rather be spent on firms in region (b) or (c) in figure 1. It is by considering this trade-off that the present considerations differ most from the analysis of incomplete enforcement of environmental taxes, as carried out by Macho-Stadler and Perez-Castrillo (2006). Generally, for any two projects with probability of verification $\beta_{1}$ and $\beta_{2}$, a marginal shift $\delta$ in monitoring effort from project 1 to project 2 is (weakly) efficient if:

$$
\begin{aligned}
& -f\left(\beta_{1}\right) \frac{\partial x\left(\alpha_{1} \beta_{1}\right)}{\partial \alpha_{1}} \delta+f\left(\beta_{2}\right)\left(\frac{\partial x\left(\alpha_{2} \beta_{2}\right)}{\partial \alpha_{2}}\right) \frac{f\left(\beta_{1}\right)}{f\left(\beta_{2}\right)} \delta>(\geq) 0 \\
& \text { with } \\
& x(\alpha \beta)= \begin{cases}\bar{e}-\underline{e}, & \text { for } \alpha \beta=0 \\
\bar{e}-e(\alpha \beta), & \text { for } 0<\alpha \beta<\frac{p}{p+\theta^{\prime}\left(\bar{e}-e^{*}\right)} \\
z(\alpha \beta)-e^{*}, & \text { for } \frac{p}{p+\theta^{\prime}\left(\bar{e}-e^{*}\right)} \leq \alpha \beta \leq \frac{p}{p+\theta^{\prime}(0)} \\
0, & \text { for } \frac{p}{p+\theta^{\prime}(0)}<\alpha \beta \leq 1\end{cases}
\end{aligned}
$$

Note that the rational regulator will never increase monitoring pressure for firms which are already in full compliance. Consequently, the case $\alpha \beta>\frac{p}{p+\theta^{\prime}(0)}$ will never occur. Furthermore, for positive $\alpha$, the case $\alpha \beta=0$ can only occur if $\beta$ is zero. 
Choosing $\alpha=0$ for this case is straightforward. Hence, identifying an efficient auditing policy through condition (14) mainly involves a trade-off in monitoring pressure between the two regions (b) and (c). This requires the comparison of the respective slopes of $e(\alpha)$ and $z(\alpha)$. As both of these functions result from the optimizing behavior of the project participants in response to the ex ante communicated $\alpha$ for each project type, these slopes can be derived from (4) and (5) by applying the Implicit Function Theorem. The corresponding partial derivatives are:

$\frac{\partial e}{\partial \alpha}=\beta \cdot e^{\prime}(\alpha \beta)=\beta \cdot \frac{p+\theta^{\prime}(\bar{e}-e(\alpha \beta))}{c^{\prime \prime}(e(\alpha \beta))+\alpha \beta \theta^{\prime \prime}(\bar{e}-e(\alpha \beta))}$, for $\left.\alpha \beta \in\right] 0, \frac{p}{p+\theta^{\prime}\left(\bar{e}-e^{*}\right)}[$

and

$\frac{\partial z}{\partial \alpha}=-\beta \cdot z^{\prime}(\alpha \beta)=-\frac{p+\theta^{\prime}\left(z(\alpha \beta)-e^{*}\right)}{\alpha \theta^{\prime \prime}\left(z(\alpha \beta)-e^{*}\right)}$, for $\alpha \beta \in\left[\frac{p}{p+\theta^{\prime}\left(\bar{e}-e^{*}\right)}, \frac{p}{p+\theta^{\prime}(0)}\right]$

Hence, as it is to be expected, within both regions (b) and (c) the amount of overreported reductions $x=z-e$ is decreasing in the overall probability of discovery. As both slopes are a function of the penalty $\theta(x)$, and $\frac{\partial e}{\partial \alpha}$ additionally depends on the abatement costs $c(e)$, identifying the optimal auditing policy for each $\beta$-type firm is only possible if additional assumptions are made. However, even the quite general forms of (15) and (16) allow to derive the following proposition.

Proposition 3. Under a limited budget $B<\bar{B}$, optimal monitoring policy implies that there exists a threshold $\beta_{l}(B)>0$, such that the regulator chooses $\alpha=0$ for $\beta \leq \beta_{l}(B)$. Projects with $\beta>\beta_{l}(B)$ will always be monitored with $\alpha>0$.

Proof: See mathematical appendix.

Proposition 3 allows a first interesting insight in the optimal distribution of audit pressure. It states that a budget-constrained rational regulator will never audit those projects that are most difficult to monitor, while all other projects will always be monitored with positive probability. It is, however, not possible under the given assumptions to make any further propositions on the intensity of monitoring pressure for those projects with levels of verifiability lying above that threshold. Hence, further insights can only be gained if the analysis is restricted by making additional assumptions. Yet, before turning to these assumptions it is useful to first introduce some simplifications in notation. Denote with $q$ the marginal penalty to be paid if project participants are caught overreporting by the amount $x(\alpha \beta)$. Hence:

$$
q(x) \equiv p+\theta^{\prime}(x)
$$

Further we define $\rho$ as the ratio of the marginal penalty to its first derivative.

$$
\rho(x) \equiv \frac{q(x)}{q^{\prime}(x)}
$$

Taking these changes in notation into account we make the following assumption. 
Assumption 1. For $x=z-e$, with $e \in[\underline{e} ; \bar{e}]$ and $z \in[\tilde{e}, \bar{e}]$ where $\tilde{e}$ is the actual level of emission reductions achieved by the project, assume that $\rho(x)$ is weakly increasing in $x$. Hence:

$$
\left(\frac{q(x)}{q^{\prime}(x)}\right)^{\prime} \geq 0 \Longleftrightarrow q^{\prime \prime}(x) \leq \frac{q^{\prime}(x)^{2}}{q(x)}
$$

Note that by the assumptions initially made in section 3, both, $q(\cdot)$ and $q^{\prime}(\cdot)$ are strictly larger than zero. Hence assumption 1 is, for example, fulfilled in the case of a polynomial penalty function of the form $\theta(x)=m \cdot x^{n}+\sum_{i=0}^{n-1} a_{i} \cdot x^{i}$, with $m>0$, $a_{i} \geq 0$, and $n \geq 2$. As shown in the mathematical appendix, Assumption 1 is a sufficient condition for both, $e^{\prime}(\alpha)$ and $-z^{\prime}(\alpha)$, to be decreasing in $\alpha$ for any $\beta>0$. This allows us to identify two other thresholds which are important for determining the optimal monitoring policy. The nature and conditions for existence of these thresholds are stated within within the following proposition.

Proposition 4. Under Assumption 1 and given a limited budget $B<\bar{B}$, the optimal monitoring policy of the regulator is characterized by the following:

(i) For $\beta_{l}(B)<1$, and if

$$
\beta_{l}(B)<\frac{z^{\prime}(\hat{\alpha}(1))}{e^{\prime}(0)}=\frac{q\left(\bar{e}-e^{*}\right)}{p} \cdot \frac{q\left(\bar{e}-e^{*}\right) \cdot c^{\prime \prime}(\underline{e})}{q^{\prime}\left(\bar{e}-e^{*}\right) \cdot q(\bar{e}-\underline{e})}
$$

there exists a threshold $\beta_{m}(B)>\beta_{l}(B)$ such that $z<\bar{e}$ for all $\beta>\beta_{m}(B)$. The value of $\beta_{m}(B)$ is non-decreasing in $B$. Furthermore, for all cases where $\beta_{l}(B)$ is strictly larger than $\frac{z^{\prime}(\hat{\alpha}(1))}{e^{\prime}(0)}$, the value of $\beta_{l}(B)$ is non-increasing in $B$.

(ii) For $\beta_{l}(B)<1$, and if

$$
\beta_{l}(B)<\frac{z^{\prime}(\check{\alpha}(1))}{e^{\prime}(0)}=\frac{q(0)}{p} \cdot \frac{q(0) \cdot c^{\prime \prime}(\underline{e})}{q^{\prime}(0) \cdot q(\bar{e}-\underline{e})}
$$

there exists a threshold $\beta_{h}(B)$, such that the regulator induces $z=e$ for firms with $\beta \geq \beta_{h}(B)$. The value of $\beta_{h}(B)$ is non-increasing in $B$.

(iii) For any $B_{0}$ for which $\beta_{l}\left(B_{0}\right)$ fulfills condition (17) (condition (18)), the condition is also fulfilled for any $\beta_{l}(B)$, for which $B>B_{0}$.

Proof: See mathematical Appendix.

Proposition 4 states the conditions for the existence of the ranges (c) and (d) under a limited budget. The budget constraint determines in fact the level of $\beta_{l}$ - the threshold from which on the regulator prefers to refrain from monitoring the projects. The existence of this threshold $\beta_{l}$ is a precondition for the existence of the other two thresholds defined in the proposition. In fact, as is shown in the mathematical appendix, the conditions for the existence of these additional thresholds are derived through the comparison of the slopes of $x=z-e$ for the highest $\beta$-values of the set of monitored projects, i.e. $\beta=1$, and the set of non-monitored projects, i.e. $\beta_{l}$. Note that for any given combination of certificate price $p$, abatement cost 
function $\mathrm{c}(\mathrm{e})$, and penalty function $\theta(z-e)$ the right hand sides of (17) and (18) are constant. As, under the given assumptions, the regulator has full information on these determinants, the existence of both thresholds is unambiguous once the lower threshold level $\beta_{l}(B)$ is known.

However, the behavior of $\beta_{l}(B)$ with respect to increases (decreases) in B might be ambiguous. While it is safe to say that $\beta_{l}(B)$ decreases for large enough increases in the budget $B$, this does not necessarily hold at the margin. Given that $x(\alpha \beta)$ features a 'jump' at $\alpha \beta=\frac{p}{p+\theta^{\prime}\left(\bar{e}-e^{*}\right)}$, a small increase in budget might lead to abrupt changes in the audit strategy. Assume, for example, that $B$ is marginally smaller than $\hat{\alpha}(1)$. Assume further that the gain from a decrease of $z$ in $z(\hat{\alpha}(1))$ for $\beta=1$ is larger than the sum of reduced overreporting for all other projects currently monitored. In this case an additional marginal increase in budget, would-according to condition (14)-lead to a decrease in overall monitored projects in favor of an increase in monitoring pressure for projects with $\beta=1$. While such discontinuities in $\beta_{l}(B)$ cannot be excluded, proposition 4 (iii) ensures that the upper threshold values continue to exist if the monitoring budget is increased. In fact, as is shown in the mathematical appendix the only reason for which the number of monitored projects might be reduced with an increase in $B$, is that the two other threshold values $\beta_{m}$ and $\beta_{h}$ are decreased. Hence, there exists a trade-off between increasing the compliance of easy-to-verify projects or weakly increasing overall monitored projects.

Once the behavior of $\beta_{l}(B)$ is understood, the threshold conditions formulated within proposition 4 are quite intuitive. The first factor in conditions (17) and (18) represents the ratio of the marginal punishment and the marginal gain, i.e. the price of one certificate, for a project facing the probability of discovery inducing a report right at the respective threshold level. The larger this ratio, the more likely is the existence of the respective threshold under a limited budget. This relationship is plausible. If the power of the incentive to further reduce overreporting or to fully comply is high it is, ceteris paribus, rational to actually induce these levels of compliance. Note that given the definition of the marginal penalty the first factor in both conditions is always larger than 1.

The second factor within conditions (17) and (18) is also quite intuitive. It represents in fact the ratio of the marginal increase in marginal abatement costs at $\beta_{l}$ to the marginal decrease in marginal punishment at the respective threshold level (i.e. $\beta_{m}$ or $\beta_{h}$ ), each set in relationship to the marginal punishment at the same level of overreporting. Note that an increase in marginal abatement costs lowers the incentive to increase the actual emission reductions, while an increase in the marginal penalty will raise this incentive. Consequently, the ratio represents a measure for the intensity of the achievable incentives at $\beta_{l}$ compared to the achievable incentives at the threshold level considered, i.e. $\beta_{m}$ or $\beta_{h}$. The harder it is to incentivize a change in behavior at $\beta_{l}$ the more likely it is that the regulator prefers to increase auditing pressure for projects with a higher degree of verifiability.

While proposition 4 provides interesting insights on the conditions under which the rational regulator decides to incentivize easier-to-verify projects toward full compliance, it is not suitable for identifying how a given budget should be optimally attributed to the different project types. As this is probably the most eminent question in the context of budget-constrained monitoring, we will present a few interesting insights to that matter in the following section. 


\subsection{Optimal Monitoring Pressure under a given Budget}

The regulator interested in the minimization of overall overreporting needs to decide on the optimal auditing pressure for each $\beta$-type project. Evidently, the optimal monitoring strategy depends on the specific functional forms of the penalty function, the abatement cost function, and the price of the certificate $p$. A specification of these determinants would, however, imply a considerable loss of generality. For this reason, we will rather present those features of an optimal monitoring strategy, which can be gained from the general model. As is shown in the mathematical appendix the assumptions made so far are sufficient to allow for several important insights on the optimal monitoring pressure $\alpha$ for specific ranges of $\beta$-type projects. These insights are merged within the following proposition.

Proposition 5. Under assumption 1 and if there exists a threshold value $\beta_{m}$ the following holds:

(i) For $\beta_{0}<\beta_{m}$ and if $\beta_{0} \rightarrow \beta_{m}$ then $\alpha\left(\beta_{0}\right)<\alpha\left(\beta_{m}\right)$

(ii) If

$$
\frac{p}{q\left(\bar{e}-e^{*}\right)}<\frac{q\left(\bar{e}-e^{*}\right) \cdot c^{\prime \prime}(\underline{e})}{q^{\prime}\left(\bar{e}-e^{*}\right) \cdot q(\bar{e}-\underline{e})}
$$

all projects with $\beta<\beta_{m}(B)$ will be monitored with probability $\alpha=0$.

(iii) For $\beta \in\left[\beta_{m}, 1\right]$ the monitoring probability $\alpha$ is strictly decreasing in $\beta$.

(iv) For $\beta \in] \beta_{l}, \beta_{m}$ [ the sign of $\frac{d \alpha}{d \beta}$ is ambiguous under the given assumptions. Yet, the following is true:

(a) if $c(\cdot)$ and $\theta(\cdot)$ are quadratic functions a sufficient condition for $\alpha(\beta)$ being strictly increasing (decreasing) for $\beta_{l}<\beta<\beta_{m}$ is:

$$
\left.c^{\prime \prime}(e)>(<) \alpha \beta \cdot \theta^{\prime \prime}(\bar{e}-e) \forall e \in\right] \underline{e} ; e^{*}[
$$

(b) if $\alpha(\beta)$ is strictly decreasing for $\beta_{l}<\beta<\beta_{m}$ and if $c^{\prime \prime}(e)$ is close to zero, the optimal $\alpha$ for $\beta$ close to $\beta_{l}$ is larger than for any $\beta \geq \beta_{m}(B)$.

Proof: See mathematical Appendix.

Proposition 5 (i) implies that the optimal audit pressure at the threshold level $\beta_{m}-$ from which on the projects will always achieve the optimal reductions-is strictly larger than for projects with a slightly lower level of $\beta$. In fact, the function of optimal audit pressure $\alpha(\beta)$ features a discontinuity at $\beta_{m}$, involving an upwards shift of the optimal audit pressure at this point.

Proposition 5 (ii) states the condition for which the threshold level $\beta_{l}$ for positive audit pressure is only marginally lower than the threshold for projects achieving the optimal level of emission reductions. Consequently, in such a case, there will be no projects situated within the (b)-range of figure 1 . Condition (19) is quite intuitive. At the left-hand side is the ratio of marginal gains and punishment from misreporting one unit for projects of type $\beta_{m}$. Note that this ratio is always smaller than one. The right hand side of condition (19) consists of the already introduced incentive measure comparing the strength of incentives at $\beta_{l}$ and $\beta_{m}$. The measure is larger than one if a marginal change in punishment $\beta_{l}$ would lead to a larger change in marginal abatement costs than the decrease it would yield in marginal punishment 
at $\beta_{m}$. Hence, proposition 5 (ii) states that the regulator refrains completely from monitoring projects with $\beta<\beta_{m}$ if such monitoring would lead to a lower change in overreported emissions than could be achieved with incentivizing a decrease in $z$ for projects with $\beta \geq \beta_{m}$.

A very important result with respect to the optimal monitoring strategy is implied within proposition 5 (iii). In fact, it states that independently of the existence of the upper threshold level $\beta_{h}$ the regulator chooses to reduce the audit pressure with increasing verifiability. Hence, even if it is possible to incentivize projects with high $\beta$ to fully comply, the regulator might decide to use his monitoring budget to rather increase pressure on the projects with a lower degree of verifiability.

The behavior of $\alpha(\beta)$ for a decrease in $\beta$ for $\beta_{l}<\beta<\beta_{m}$ is more ambiguous. While it is clear from proposition 5 (i) that the optimal audit pressure feature a sudden decrease if $\beta$ decreases further than $\beta_{m}$, the given assumptions are insufficient to determine if $\alpha(\beta)$ is increasing or decreasing for projects with a verifiability lower than $\beta_{m}$. Proposition 5 (iv) sheds some light on the optimal monitoring policy for projects with such an intermediate degree of verifiability. Under the standard assumption of cost and penalty functions being quadratic, optimal audit pressure in this range of verifiability depends in principle on how strict the punishment is compared to the respective abatement costs. For a relatively lax quadratic penalty function, the first inequality in condition (20) implies that an increase in actual emissions would lead to a larger increase in marginal abatement costs than it would decrease the marginal penalty. Hence, under these conditions, the change in incentive to misreport is larger than the change in disincentive stemming from the punishment. As a consequence, it would be inefficient to increase audit pressure for lower degrees of verifiability, as the gains in compliance would be smaller than for projects with higher degrees of verifiability. The opposite is true if the punishment function is sufficiently stricter than the abatement costs function, which is reflected within the bracketed inequality in condition (20). In this case, the difference in incentive change increases with decreasing $\beta$. Hence, simply spoken, if the punishment is sufficiently large, it becomes optimal for the regulator to exercise an ever higher audit pressure for lower levels of verifiability within the range of $\beta_{l}<\beta<\beta_{m}$. Interestingly, if the cost function is close enough to linear, the audit pressure will be largest for projects with a $\beta$ close to $\beta_{l}$. Hence, the optimal audit policy of the regulator crucially depends on the punishment scheme that is foreseen by the legislative body.

In order to summarize the most important findings implied by the propositions 3 to 5 , it is useful to visualize the optimal monitoring policy chosen by the regulator under different setups. In figure 2 we hence depict two different situations for both of which the budget level $\mathrm{B}$ is smaller than $\bar{B}$ but large enough to ensure the existence of the threshold levels $\beta_{m}$ and $\beta_{h}$. Consequently, in both of these setups all possible levels of compliance (or misreporting) are realized. The depicted situations 1 and 2 differ, in fact, only with respect to the form of the abatement cost function and its relationship to the penalty function, leading to the different monitoring strategies $\alpha_{1}(\beta)$ and $\alpha_{2}(\beta)$. We first describe the qualitative relationships in monitoring pressure which is common to both of these setups. As stated in proposition 3, in all cases the range of projects with the lowest degree of verifiability will not be monitored at all. At first sight, this might be considered as counter-intuitive as it could be argued that projects with low verifiability would require a high level of monitoring. However, given that the regulator is to maximize overall emission 
reductions under a constrained budget, it turns out to be more efficient to completely ignore projects below the threshold $\beta_{l}$. Note that this would also be the case for an environmental tax scheme as analyzed by Macho-Stadler and Perez-Castrillo (2006).

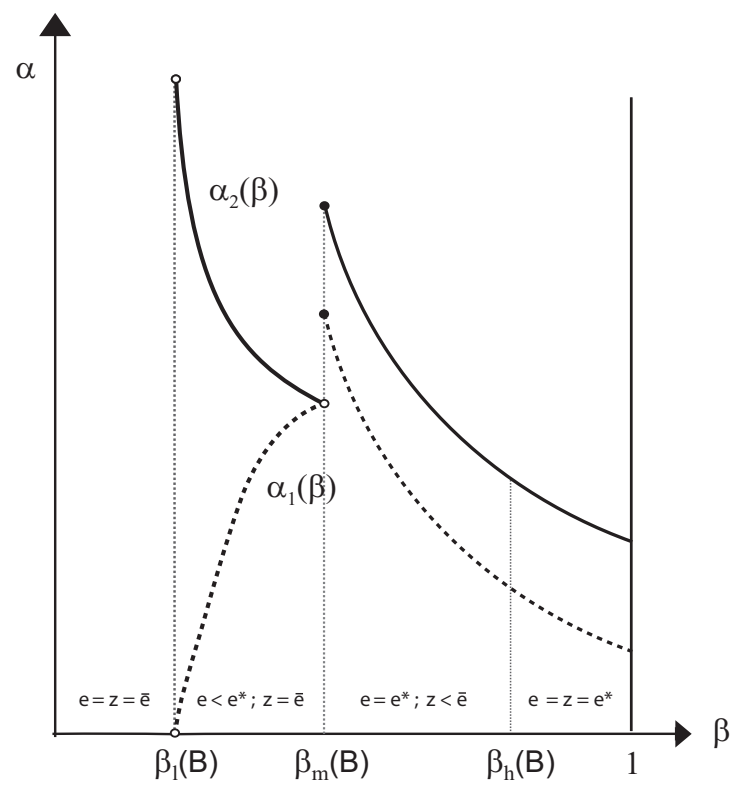

Figure 2: Optimal auditing pressure for different assumptions on abatement costs

Furthermore, in both situations depicted in figure 2, the optimal auditing pressure features the upwards jump at $\beta_{m}$, stated in proposition 5 (i). From this point onwards optimal monitoring pressure is always strictly decreasing in the degree of verifiability, as stated in proposition 5 (iii). Hence, the easier the projects are to verify, the lower is the optimal monitoring probability for the respective project types. This is particularly intuitive for those projects that are in complete compliance, i.e. projects for which verifiability is larger or equal to $\beta_{h}$. As these projects report truthfully, an increase in monitoring probability would not lead to further increases in overall emission reductions. For projects with an efficient emission level but an untruthful report, situated between $\beta_{m}$ and $\beta_{h}$, the relationship in monitoring intensity is more interesting. As already stated, with a credit-based system a regulator interested in reducing overall emissions needs to take into account both, reductions in overreporting through the report $z$, as well as actual emissions $e$. Hence, the fact that this trade-off results in an unambiguous qualitative relationship for monitoring projects situated in field (c) of figure 1 is quite an important finding. Another interesting result is the fact that for cases for which condition (19) holds, the monitoring pressure for all $\beta$ lower than $\beta_{m}$ would be zero. Consequently, the optimal monitoring function would only consist of the right hand branch of both depicted functions if the curvature of the abatement cost function were large enough. Hence, if the increase in marginal abatement cost is particularly large, the optimal monitoring would require to entirely refrain from monitoring all projects that would not yield the efficient level of reductions $e^{*}$.

The only ambiguity that exists in the qualitative relationship of optimal monitoring is with respect to levels of verifiability situated between thresholds $\beta_{l}$ and $\beta_{m}$. As stated in proposition 5 (iv), optimal monitoring might either increase or 
decrease in the degree of project verifiability. This ambiguity can be resolved if functional forms for the penalty and the abatement costs are specified. The optimal monitoring strategy $\alpha_{1}(\beta)$, depicted in figure 2 , applies to a situation where the increase and curvature of the quadratic cost function is relatively large compared to the penalty function. This represents a case for which the unbracketed version of condition (20) holds. Under such circumstances, the optimal monitoring probability is strictly increasing in $\beta$ for the respective range. In contrast, situation 2 represents a case where the abatement cost function is close to linear, while the penalty function is comparatively strict. Hence, for $\beta_{l}<\beta<\beta_{m}$, the monitoring function $\alpha_{2}(\beta)$ is decreasing and reaches its largest values for $\beta$ close to $\beta_{l}$, as stated in proposition 5 (iv)(b). The differentiation between these two cases hence showcases that the regulator's optimal strategy not only depends on his budget, but also on the relationship between abatement costs and punishment.

While the abatement costs are usually beyond the control of a regulatory framework, the penalty can be-at least in principle-easily adjusted. However, in the real world it is likely that there exist legal and political constraints with respect to the level of punishment. Clearly, the rule of law requires commensurability in legal sanctions, such that the set of applicable penalties is considerably reduced. As far as the CDM is concerned, it is also to note that sanctions beyond the rules of private law would have to be accepted within the COPs. The present Kyoto rules do not explicitly specify a penalty schedule for over-reporting of emissions. Yet, the model results presented above emphasize the importance of stipulating specific sanctions in order to assure a certain level of effectiveness for the CDM. However, as with all Kyoto rules, provisions on sanctions for the CDM are subject to the constraints of political feasibility. Depending on whether these constraints allow for lax or stricter sanctions the CDM Executive Board would have to decide on a monitoring strategy which rather resembles to $\alpha_{1}(\beta)$ or $\alpha_{2}(\beta)$ in figure 2 .

\subsection{Regulation with Project Admission}

The CDM has long been and in fact remains a matter of political dispute within the Kyoto negotiations. Even in the potential buyer countries, the use of Certified Emission Rights for meeting the Kyoto targets is not undisputed, as some observers still challenge the morality of reducing emissions in third-world countries. As a consequence, the sudden discovery of large scale fraud within a CDM project would significantly undermine the credibility of the whole Kyoto emissions trading regime as an instrument to achieve emission reductions. Hence, it is plausible that the architects of the Mechanism might want to reduce the risk of discovery of fraud. As the above-presented model results have shown this is unlikely to be effectively achievable with project monitoring alone.

In this context it might be interesting to further reduce the potential of overreporting by adjusting the rules for project admission. Within the CDM for example, the regulator can refuse the admission of a project if the Project Design Document or the proposed Baseline Method do not correspond to the specified standards. ${ }^{5} \mathrm{It}$ is hence quite plausible that project admission standards could also include a minimum level of verifiability. Such a standard could apply to both sources of overreporting identified above, the baseline as well as the verification of project emissions.

\footnotetext{
${ }^{5}$ The probability of rejection for a submitted CDM project is about 5 percent. (UNEP/Risoe (2009))
} 
The above-presented model results can be used to gain valuable insights for determining sensible cut-off levels for project admission. In the context of this model, the Kyoto architects would have to decide on a maximum tolerable level of opportunistic misreporting given a specified budget for the CDM Executive Board. It is, for example, conceivable that the regulator implements a 'no tolerance' constraint, which would exclude all projects with a verifiability lower than the minimal $\beta_{h}(B)$. In this case all projects would be in perfect compliance. Another intuitively sensible cut off-level would be $\beta_{m}(B)$, implying that all projects will achieve the optimal level of emission reductions, while cheating within the reported level would not be perfectly deterred. Based on the experiences made up to the present, project admission standards would have to be made more stringent until only projects with a verifiability larger or equal to the respective threshold remain.

Note that, for the time being, the justification for the inclusion of a minimum level of verifiability within the project admission standards can only be based on the political justification depicted above. In light of the fact that there exists no consensus on the allocatively optimal level of emission reductions, determining a specific cut-off level based on welfare considerations would be particularly problematic. Hence, as long as the exact level of the social cost of carbon is still disputed, the optimal regulation under incomplete enforcement remains just as undetermined as the optimal level of abatement.

\section{Conclusion}

The model presented within this paper allows some interesting insights into the nature of optimal monitoring for credit-based systems, like the Clean Development Mechanism. It was shown that under these circumstances even with an unlimited monitoring budget, overreporting of reductions can not be completely disincentivized. The most interesting results are, however, achieved under the more realistic assumption of a budget constraint. For this purpose, the analysis above has been restricted to a specific class of punishment functions, which includes polynomial penalties.

While under an unlimited budget all projects with positive verifiability will be monitored, the situation significantly changes under the assumption of a budget constraint. For this case, it is shown that a rational regulator will completely refrain from monitoring those projects that are most difficult to verify. Furthermore, for those projects that are induced to achieve the optimal level of reductions the monitoring pressure reduces with increasing verifiability of the projects. Both results are in line with the findings by Macho-Stadler and Perez-Castrillo (2006) who analyze incomplete enforcement of emission taxes. However, unlike emission taxes, the general principle of credit-based emission trading systems implies that the regulator cannot refrain to simply maximize the actual emission reductions but needs also to reduce the level of overstatement within the projects' reported reductions. This is due to the fact that certificates issued on the basis of the reports will be used to offset actual emissions elsewhere. Hence, contrary to the the tax case, the regulator needs to minimize the overall level of overreporting.

Due to this difference in the objective function of the regulator, the optimal monitoring strategy derived above significantly differs from those proposed in the context of emission taxes or a cap-and-trade system. First, given a large enough budget, the regulator will induce full compliance within the range of the easiest-to-verify 
projects. Second, with decreasing verifiability, the optimal audit pressure features a 'jump' downwards as soon as the region of those projects is reached, for which the regulator decides not to incentivize the optimal level of emission reductions. Within this region of lower verifiability it depends on the exact interaction of incentives and disincentives to increase compliance. The disincentives are determined through the abatement cost function while the incentives stem from the punishment, which is assumed to be increasing and convex in the overreported amount. Depending on the functional forms of these two functions it is either possible that optimal auditing pressure increases or decreases with a reduction in verifiability. Third, due to the above-described discontinuity in auditing pressure, it might be possible that a small increase in budget leads to a decrease in audited projects. A large enough budget rise, however, will broaden the set of projects being monitored.

The monitoring budget, in fact, determines also the number of projects that are situated within the different regions of compliance. This leads to the interesting insight that the regulator could include a specific level of verifiability within the project admission criteria. This would guarantee that a maximum amount of overreporting will never be exceeded under a given budget. The latter result might be especially interesting in the case of the Clean Development Mechanism, which is by far not undisputed. The discovery of large fraudulent overreporting within that mechanism would certainly entail a large damage to its credibility. 


\section{Mathematical Appendix}

\section{Sufficient conditions for (4) and (5) denoting a minimum:}

$$
\begin{aligned}
& \frac{\partial^{2} C}{\partial e^{2}}=c^{\prime \prime}(e)+\alpha \beta \theta^{\prime \prime}(z-e)>0 \\
& \quad \frac{\partial^{2} C}{\partial z^{2}}=\alpha \beta \theta^{\prime \prime}(z-e)>0 \\
& \quad \frac{\partial^{2} C}{\partial e^{2}} \frac{\partial^{2} C}{\partial z^{2}}-\left[\frac{\partial^{2} C}{\partial e \partial z}\right]^{2}=\alpha \beta c^{\prime \prime}(e) \theta^{\prime \prime}(z-e) \geq 0 \\
& \quad \Longrightarrow \text { Solution for the first order conditions (4) and (5) denote a minimum. }
\end{aligned}
$$

\section{Proof of Proposition 1:}

Note that $e^{0}$ is, by definition, strictly positive. For $\alpha \beta=0$, Equation (3) becomes $c(e)-p z$ which is evidently minimized by $e^{0}=\underline{e}$ and $z^{0}=\bar{e}$, that is we are in region (a).

For the rest of this proof it is supposed that $\alpha \beta>0$. From equation (5) follows that the report $z^{0}$ only represents an interior solution if and only if $\alpha \beta \theta^{\prime}(0)<$ $p(1-\alpha \beta)<\alpha \beta \theta^{\prime}\left(\bar{e}-e^{0}\right)$.

When $p(1-\alpha \beta) \geq \alpha \beta \theta^{\prime}(0)$ the corner solution is $z^{0}=e^{0}$, the firm's report is honest. Under these conditions, it follows from (4) that $e^{0}=e^{*}$. We are, thus in region $(d)$.

When $\alpha \beta \theta^{\prime}\left(\bar{e}-e^{0}\right) \geq p(1-\alpha \beta)$ the firm reports the maximum believable emissions reduction, i.e. $z^{0}=\bar{e}$. The firm thus chooses $e^{0}$ satisfying (4) for $z^{0}=\bar{e}$, i.e. $e^{0}$ satisfies (6). This pair, $z^{0}=\bar{e}$ and $e^{0}$ satisfying (6), is indeed a candidate for maximization if and only if $\alpha \beta \theta^{\prime}\left(\bar{e}-e^{0}\right) \geq p(1-\alpha \beta)$ for the proposed $e^{0}$. Given (6) the previous inequality is equivalent to $p \geq c^{\prime}\left(\bar{e}-e^{0}\right)$, i.e. $e^{0} \geq e^{*}$. Thus, we are in region (b). When both, emission reductions and report are interior, then adding (4) and (5) this leads to $p=c^{\prime}\left(\bar{e}-e^{0}\right)$, i.e. $e^{0}=e^{*}$. The optimal report $z^{0}$ in this region is given by (5) for $e^{0}=e^{*}$, thus it is given by equation (7).

\section{Proof of Proposition 3:}

For $\beta \rightarrow 0$, $\frac{\partial e}{\partial \alpha}$, given by equation (15), approaches 0 as well, as $e^{\prime}(\alpha \beta)$ is never infinitely large for $e \in[\underline{e}, \bar{e}]$. Hence, according to condition (14), for $\beta_{1}<\beta_{2}$ and $\beta_{1}$ small enough, a decrease in $\alpha_{1}$ is always efficient as long as $\alpha_{1}>0$. Furthermore, it is easy to check that for $\alpha=0$ the function $e^{\prime}(\alpha)$ is increasing in $\beta$. Hence, it follows from condition (14) that all projects with $\beta>\beta_{l}(B)$ are monitored with positive probability.

\section{Proof of Proposition 4}

In order to prove proposition 4 we first state and prove the following two Lemmas.

\section{Lemma A.1:}

For any pair of projects with $\beta_{1}$ and $\beta_{2}$ for which the probability of discovery is $\alpha_{i} \beta_{i} \in\left[\frac{p}{p+\theta^{\prime}\left(\bar{e}-e^{*}\right)} ; \frac{p}{p+\theta^{\prime}(0)}\right]$, optimal monitoring requires:

$$
\frac{\partial x_{1}}{\partial \alpha_{1}}=\frac{\partial x_{2}}{\partial \alpha_{2}}
$$




\section{Proof of Lemma A.1}

Lemma A.1 follows directly from the efficiency condition (14). For any two firms with verifiability $\beta_{1}$ and $\beta_{2}$ monitored with $\alpha_{1}$ and $\alpha_{2}$, condition (14) requires that at the optimum a decrease in $\alpha_{1}$ in order to increase $\alpha_{2}$ would lead to

$$
f\left(\beta_{1}\right) \delta\left[-\frac{\partial x_{1}}{\partial \alpha_{1}}+\frac{\partial x_{2}}{\partial \alpha_{2}}\right] \leq 0, \text { where } x_{i}=z_{i}-e_{i}
$$

As for any pair of projects with $\left.\left.\alpha_{i} \beta_{i} \in\right] 0 ; \frac{p}{p+\theta^{\prime}(0)}\right]$ the two projects can be alternatingly defined as project 1 and 2 , condition (22) only holds if $\frac{\partial x_{1}}{\partial \alpha_{1}}=\frac{\partial x_{2}}{\partial \alpha_{2}}$.

Next we show that Assumption 1 guarantees that $-z^{\prime}(\alpha)$ and $e^{\prime}(\alpha)$ are decreasing in $\alpha$. The curvature of function $z(\alpha)$ for any given $\beta$ is given by:

$$
-z^{\prime \prime}(\alpha)=\frac{-2 q_{z}(x) q_{z}^{\prime}(x)+\frac{q_{z}^{\prime \prime}(x) q_{z}^{2}}{q_{z}^{\prime}}}{\alpha^{2} q_{z}^{\prime}(x)^{2}}
$$

Hence, $-z^{\prime \prime}(\alpha)<0$ iff:

$$
\begin{gathered}
q^{\prime \prime}(x)-2 \cdot \frac{q^{\prime}(x)^{2}}{q(x)}<0, \quad \text { with } x \in\left[0,\left(\bar{e}-e^{*}\right)\right] \\
e^{\prime \prime}(\alpha)=\frac{-2 q_{e} q_{e}^{\prime}-\frac{q_{e}^{2}\left(c^{\prime \prime \prime}(e)-\alpha \beta q_{e}^{\prime \prime}\right)}{c^{\prime \prime}(e(\alpha))+\alpha \beta q_{e}^{\prime}}}{\left(\frac{1}{\beta} c^{\prime \prime}(e(\alpha))+\alpha q_{e}^{\prime}(x)\right)^{2}}, \quad \text { with } x \in\left[0,(\bar{e}-\underline{e}),\left(\bar{e}-e^{*}\right)[\right.
\end{gathered}
$$

Hence, $e^{\prime \prime}(\alpha)<0$ iff:

$$
q^{\prime \prime}(x)-2 \cdot \frac{q^{\prime}(x)^{2}}{q(x)}<2 \rho(x) c^{\prime \prime}(\bar{e}-x)+c^{\prime \prime \prime}(\bar{e}-x)
$$

Assumption 1 is, thus, a sufficient condition for $e^{\prime}(\alpha)$ and $-z^{\prime}(\alpha)$ to be decreasing in $\alpha$. Hence, the following Lemma A.2 is straightforward.

\section{Lemma A.2:}

Under Assumption 1 the following holds for any $\left.\left.\beta_{0} \in\right] 0,1\right]$ monitored with probability $\left.\left.\alpha_{0} \in\right] 0,1\right]$

$$
\left.e^{\prime}\left(\alpha_{0}\right)=\beta_{0} \cdot e^{\prime}\left(\alpha_{0} \beta_{0}\right)<\beta_{0} \cdot e^{\prime}(0), \text { for } \alpha \beta \in\right] 0, \frac{p}{p+\theta^{\prime}\left(\bar{e}-e^{*}\right)}[
$$

Based on Lemmas A.1 and A.2 we can now proceed with the proof of Proposition 4. 


\section{Proof of Proposition 4(i)}

Consider a project 1 with $\beta_{1}=1$. In order to incentivize $z<\bar{e}$ for this project, the regulator must set $\alpha_{1}>\hat{\alpha}(1)$. As, under assumption $1,-z^{\prime}(\alpha)$ is strictly decreasing in $\alpha$, the corresponding level of $-z^{\prime}(\alpha)$ for $\beta_{1}=1$ must fulfill

$$
-z^{\prime}\left(\alpha_{1}\right)<-z^{\prime}(\hat{\alpha}(1))=\frac{\left(p+\theta^{\prime}\left(\bar{e}-e^{*}\right)\right)^{2}}{p \theta^{\prime \prime}\left(\bar{e}-e^{*}\right)}
$$

If the monitoring Budget is large enough, such that optimal monitoring requires $\alpha_{1}$ for $\beta_{1}=1$ then it follows from condition (14) and Lemma A.2, that $-z^{\prime}\left(\alpha_{1}\right)>$ $\beta_{l}(B) \cdot e^{\prime}(0)$. Substituting this result into 26 yields:

$$
\beta_{l}(B) \frac{p+\theta^{\prime}(\bar{e}-\underline{e})}{c^{\prime \prime}(\underline{e})}<\frac{\left(p+\theta^{\prime}\left(\bar{e}-e^{*}\right)\right)^{2}}{p \theta^{\prime \prime}\left(\bar{e}-e^{*}\right)}
$$

This expression is equivalent to (17). Hence, if condition (17) holds, there exists at least one project, namely $\beta=1$, for which $z<\bar{e}$.

Furthermore, if (17) holds, it follows from Lemma A.1 and the fact that $z^{\prime}(\alpha)=$ $\beta \cdot z^{\prime}(\alpha \beta)$ is decreasing in $\alpha$ and continuous in $\alpha \beta$ that there exists a $\beta_{m}(B)<\beta_{1}$, such that

$$
-z^{\prime}\left(\alpha_{1}\right)=\beta_{m}(B) \cdot z^{\prime}\left(\alpha_{m} \beta_{m}\right)=\beta_{m} \cdot z^{\prime}\left(\frac{p}{p+\theta^{\prime}\left(\bar{e}-e^{*}\right)}\right)
$$

For any $\beta_{0}>\beta_{m}$, monitored with its optimal probability $\alpha_{0}$, the respective slope of the report function is $z^{\prime}\left(\alpha_{0}\right)=\beta_{0} \cdot z^{\prime}\left(\alpha_{0} \beta_{0}\right)$. As $\beta_{0}>\beta_{m}$, Lemma A.1 implies that $z^{\prime}\left(\alpha_{0} \beta_{0}\right)<z^{\prime}\left(\alpha_{m} \beta_{m}\right)$ which is equivalent to:

$$
\frac{\alpha_{0} \beta_{0}}{\alpha_{m} \beta_{m}}>\frac{\rho\left(z\left(\alpha_{0} \beta_{0}\right)-e^{*}\right)}{\rho\left(\bar{e}-e^{*}\right)}
$$

Under Assumption 1 this condition only holds if $\alpha_{0} \beta_{0}>\alpha_{m} \beta_{m}=\frac{p}{p+\theta^{\prime}\left(\bar{e}-e^{*}\right)}$. Hence, all projects larger than $\beta_{m}$ will be incentivized to a report $z_{0}$ which is strictly lower than $\bar{e}$.

An increase in $B$ is equivalent to an increase of the overall sum of available $\alpha$. As under Assumption $1 \frac{\partial e}{\partial \alpha}$ and $-\frac{\partial z}{\partial \alpha}$ are strictly decreasing in $\alpha$, an increase in $\mathrm{B}$ will equally decrease both sides of 21 for any $\beta$ monitored with positive $\alpha$. If $\beta_{m}$ exists after the increase in $B$-which is proven below-the associated slope $-z^{\prime}(\alpha)$ at $\beta_{m}$ is defined as the right hand side of (27). Hence, in order to fulfill (21), $\beta_{m}$ must decrease with an increase in $B$. It is straightforward that the same applies for $\beta_{l}$ for those cases where $\beta_{m}$ does not exist.

\section{Proof of Proposition 4(ii)}

The proof is very similiar to the proof of proposition $4(\mathrm{i})$. For $\beta=1 z=0$ if $\alpha \geq \check{\alpha}(1)$. Applying Lemmas A.1 and A.2 one can show that $\check{\alpha}(1)$ is only chosen if:

$$
\beta_{l}(B) \frac{p+\theta^{\prime}(\bar{e}-\underline{e})}{c^{\prime \prime}(\underline{e})}<\frac{\left(p+\theta^{\prime}(0)^{2}\right.}{p \theta^{\prime \prime}(0)}
$$

This expression is equivalent to (18). Hence, if condition (18) holds, there exists at least one project, namely $\beta=1$, for which $z=e$. The rest of the proof is analogous to the proof of proposition 4.4 (i) and therefore omitted here. 


\section{Proof of Proposition 4(iii)}

For all changes where $\beta_{l}(B)$ weakly decreases with $B$, the proof is trivial as condition (17) (condition (18)) will necessarily be fulfilled. The only reason for which $\beta_{l}(B)$ decreases with $B$ is that the thusly available units of $\alpha$ can be used to increase auditing pressure in the interval $\left[\beta_{m} ; 1\right]$, hence $\beta_{m}$ must exist. As $-\frac{d z}{d \alpha}$ is decreasing and continuous in $\alpha$, the same argument applies to $\beta_{h}$.

\section{Proof of Proposition 5(i)}

The result is straightforward, as

$$
\lim _{\beta_{0} \rightarrow \beta_{m}} \beta_{0} \frac{p+\theta^{\prime}\left(\bar{e}-e\left(\alpha_{0} \beta_{0}\right)\right)}{\left.c^{\prime \prime}\left(e\left(\alpha_{0} \beta_{0}\right)\right)\right)+\alpha_{0} \beta_{0} \theta^{\prime \prime}\left(\bar{e}-e\left(\alpha_{0} \beta_{0}\right)\right)}=\beta_{m} \frac{p+\theta^{\prime}\left(\bar{e}-e^{*}\right)}{c^{\prime \prime}\left(e^{*}\right)+\alpha_{0} \beta_{m} \theta^{\prime \prime}\left(\bar{e}-e^{*}\right)}
$$

will only fulfill 21 if $\alpha_{0}<\alpha_{m}$.

\section{Proof of Proposition 5(ii)}

Proposition 5(ii) is proven by contradiction. Assume that under condition (19) a project with $\beta_{0}<\beta_{m}(B)$ is monitored with positive probability. Then Lemmas A.1 and $A .2$ require

$$
\beta_{m}(B) \cdot \frac{\left(p+\theta^{\prime}\left(\bar{e}-e^{*}\right)\right)^{2}}{p \theta^{\prime \prime}\left(\bar{e}-e^{*}\right)}<\beta_{0} \cdot \frac{p+\theta^{\prime}(\bar{e}-\underline{e})}{c^{\prime \prime}(\underline{e})}
$$

As $\beta_{0}<\beta_{m}(B)$, (30) would only be true, if (19) were false.

\section{Proof of Proposition 5(iii)}

For any two projects with $\beta_{1}$ and $\beta_{2}$, with $\beta_{i} \geq \beta_{m}(B)$, Lemma A.1 implies for $\beta_{1}<\beta_{2}$ :

$$
\frac{\alpha_{2} \beta_{2}}{\alpha_{1} \beta_{1}}>\frac{\rho\left(z\left(\alpha_{2} \beta_{2}\right)-e^{*}\right)}{\rho\left(z\left(\alpha_{1} \beta_{1}\right)-e^{*}\right)}
$$

Given Assumption 1, this inequality is only true if $\alpha_{1}>\alpha_{2}$, which proves proposition 5(ii).

\section{Proof of Proposition 5(iv)}

If $\frac{\partial e}{\partial \alpha}$ strictly increases (strictly decreases) in $\beta$ then it follows from Lemma A.1 and (15) that $\alpha\left(\beta_{1}\right)$ is lower than (greater than) $\alpha\left(\beta_{2}\right)$ for any $\beta_{1}<\beta_{2}<\beta_{m}$.

Given (15) the following holds:

$$
\frac{d^{2} e(\alpha)}{d \alpha d \beta}=e^{\prime}(\alpha \beta)+\alpha \beta e^{\prime \prime}(\alpha \beta) \lessgtr 0
$$


$c^{\prime \prime}(e(\alpha \beta))^{2}-(\alpha \beta)^{2} \cdot \theta^{\prime \prime}(\bar{e}-e(\alpha \beta))^{2} \lessgtr \alpha \beta\left(p+\theta^{\prime}(\bar{e}-e(\alpha \beta))\right) \cdot\left(c^{\prime \prime \prime}(e(\alpha \beta))-\theta^{\prime \prime \prime}(\bar{e}-e(\alpha \beta))\right)$

If $c(\cdot)$ and $\theta(\cdot)$ are quadratic functions, the right hand side of (32) is zero. The rest of the proof for 5 (iv)(a) is straightforward. It remains to prove 5 (iv)(b). It follows from proposition 5 (iii) that the largest $\alpha(\beta)$ within the interval $\left[\beta_{m} ; 1\right]$ is $\alpha\left(\beta_{m}\right)$. From Lemma A.1 follows that for any monitored $\beta_{0}$ in the interval $] 0 ; \beta_{m}[$ the following holds:

$$
\beta_{m} \cdot z^{\prime}\left(\frac{p}{p+\theta^{\prime}\left(\bar{e}-e^{*}\right)}\right)=\beta_{0} \cdot \frac{p+\theta^{\prime}\left(\bar{e}-e\left(\alpha_{0} \beta_{0}\right)\right)}{c^{\prime \prime}\left(e\left(\alpha_{0} \beta_{0}\right)\right)+\alpha_{0} \beta_{0} \theta^{\prime \prime}\left(\bar{e}-e\left(\alpha_{0} \beta_{0}\right)\right)}
$$

For c"(e) close to zero and under Assumption 1 this equation will only hold if $\alpha_{0}>\alpha_{m}$ 


\section{References}

Becker, G. S.: 1968, Crime and Punishment: An Economic Approach, Journal of Political Economy 76(2), 169-217.

Cremer, H. and Gahvari, F.: 2002, Imperfect observability of emissions and secondbest emission and output taxes, Journal of Public Economics 85(3), 385-407.

Harford, J. D.: 1978, Firm behavior under imperfectly enforceable pollution standards and taxes, Journal of Environmental Economics and Management 5(1), 2643.

Harford, J. D.: 1987, Self-reporting of pollution and the firm's behavior under imperfectly enforceable regulations, Journal of Environmental Economics and Management 14(3), 293-303.

Keeler, A. G.: 1991, Noncompliant firms in transferable discharge permit markets: Some extensions, Journal of Environmental Economics and Management 21(2), 180-189.

Macho-Stadler, I.: 2006, Environmental Regulation: Choice of Instruments under Imperfect Compliance, Ufae and iae working papers, Unitat de Fonaments de I'Analisi Economica (UAB) and Institut d'Analisi Economica (CSIC).

Macho-Stadler, I. and Perez-Castrillo, D.: 2006, Optimal enforcement policy and firms' emissions and compliance with environmental taxes, Journal of Environmental Economics and Management 51(1), 110-131.

Malik, A. S.: 1990, Markets for pollution control when firms are noncompliant, Journal of Environmental Economics and Management 18(2), 97-106.

Sandmo, A.: 2002, Efficient Environmental Policy with Imperfect Compliance, Environmental and Resource Economics 23, 85-103.

Schmutzler, A. and Goulder, L. H.: 1997, The Choice between Emission Taxes and Output Taxes under Imperfect Monitoring, Journal of Environmental Economics and Management 32(1), 51-64.

Stranlund, J. K.: 2007, The regulatory choice of noncompliance in emissions trading programs, Environmental and Resource Economics 38, 99-117.

UNEP/Risoe: 2009, UNEP Risoe CDM/JI Pipeline Analysis and Database, June 1st 2009, Database and Analysis Tool, United Nations Environment Programme, RISOE Centre, Denmark. Available at: http://cdmpipeline.org/. 



\section{List of IED working papers}

- Renate Schubert, Julia Blasch, and Kristin Hoffmann (2007). Environmental Protection, Energy Policy and Poverty Reduction - Synergies of an Integrated Approach. IED Working Paper 1, ETH Zurich, Institute for Environmental Decisions, Chair of Economics.

- Robert Finger and Werner Hediger (2007). The application of robust regression to a production function comparison - the example of Swiss corn. IED Working Paper 2, ETH Zurich, Institute for Environmental Decisions, Agri-Food and Agri-Environmental Economics Group.

- Stefanie Engel and Charles Palmer (2008). "Painting the forest REDD?" Prospects for mitigating climate change through reducing emissions from deforestation and degradation. IED Working Paper 3, ETH Zurich, Institute for Environmental Decisions, Chair of Environmental Policy and Economics.

- Lasse Wallquist and Mischa Werner (2008). Carbon dioxide Capture and Storage - CCSStudie zum Entwicklungsstand von CCS in der Schweiz. IED Working Paper 4, ETH Zurich, Institute for Environmental Decisions, Chair of Consumer Behavior.

- Roland W. Scholz and Michael Siegrist (2008). Low Risks, High Public Concern? The Cases of Persistent Organic Pollutants (POPs), Heavy Metals, and Nanotech Particles. IED Working Paper 5. ETH Zurich, Institute for Envi- ronmental Decisions, Chair of Natural and Social Science Interface, Chair of Consumer Behavior.

- Astrid Zabel, Karen Pittel, Göran Bostedt, and Stefanie Engel (2009). Comparing Conventional and New Policy Approaches for Carnivore Conservation - Theoretical Results and Application to Tiger Conservation. IED Working Paper 6, ETH Zurich, Institute for Environmental Decisions, Chair of Environmental Policy and Economics.

- Astrid Zabel and Brian Roe (2009) Performance payments for environmental services: Lessons from economic theory on the strength of in centives in the presence of performance risk and performance measurement distortion. IED Working Paper 7, ETH Zurich, Institute for Environmental Decisions, Chair of Environmental Policy and Economics.

- Stefanie Engel and Charles Palmer (2009). The Complexities of Decentralization in a Globalizing World. IED Working Paper 8, ETH Zurich, Institute for Environmental Decisions, Chair of Environmental Policy and Economics.

- Michael Hartmann, Robert Huber, Simon Peter, and Bernard Lehmann (2009). Strategies to mitigate greenhouse gas and nitrogen emissions in Swiss agriculture: the application of an integrated sector model. IED Working Paper 9, ETH Zurich, Institute for Environmental Decisions, Agri-Food and Agri-Environmental Economics Group.
- Julia Blasch, Renate Schubert, Birgit Soete (2010). Grün aus der Krise Was können "grüne» Konjunkturpakete leisten? IED Working Paper 10, ETH Zurich, Institute for Environmental Decisions, Chair of Economics.

Moritz Rohling und Markus Ohndorf (2010). Prices vs. Quantities with Fiscal Cushioning. IED Working Paper 11, ETH Zurich, Institute for Environmental Decisions, Chair of Economics.

- Thomas Bernauer and Lena Maria Schaffer (2010). Climate Change Governance. IED Working Paper 12, ETH Zurich, Institute for Environmental Decisions, Chair of International Relations.

- Markus Ohndorf (2010). Optimal Monitoring for project-based Emissions Trading Systems under incomplete Enforcement. IED Working Paper 13, ETH Zurich, Institute for Environmental Decisions, Chair of Economics.

- Justin Caron and Markus Ohndorf (2010). Irreversibility and Optimal Timing of Climate Policy. IED Working Paper 14, ETH Zurich, Institute for Environmental Decisions, Chair of Economics. 
H Zurich

Institute for Environmental Decisions IED

WEH G

CH-8092 Zurich

SWITZERLAND

Phone +41446324718

Fax +41446321042

URL http://www.ied.ethz.ch 